\title{
Prevalence of nasal carriage of Staphylococcus aureus in allergic rhinitis patients and healthy controls in Thailand
}

\author{
Kanokrat Suvarnsit, ${ }^{1}$ Pattarachai Kiratisin, ${ }^{2}$ Chaweewan Bunnag, ${ }^{1}$ Pongsakorn Tantilipikorn ${ }^{1}$
}

\begin{abstract}
Background: Staphylococcus aureus has been proposed as a disease modifier of allergic rhinitis (AR) severity. Although several studies have investigated the prevalence of nasal carriage of $S$. aureus in healthy controls and AR patients, data from Thailand is scarce.
\end{abstract}

Objective: The aim of this study was to determine the prevalence of nasal carriage of S. aureus in AR patients compared with healthy controls in Thailand.

Methods: This prospective study enrolled non-AR healthy controls and confirmed AR aged 18-60 years who attended the Department of Otorhinolaryngology, Faculty of Medicine Siriraj Hospital, Mahidol University, Bangkok, Thailand during June 2013 and December 2013. To detect nasal carriage of S. aureus, nasal swab was used for specimen collection from the nasal vestibule. S. aureus prevalence was compared between groups. All AR patients were assessed for disease severity and quality of life.

Results: The 200 enrolled participants were evenly divided between the AR and healthy control groups. Nasal swab cultures were positive for $S$. aureus in 20 of 100 subjects in the healthy control group, and in 21 of 100 subjects in the AR group $(p=0.86)$. Nasal carriage of $S$. aureus was significantly more prevalent in males than in females $(p=0.01)$. None of the investigated factors were found to be significantly associated with AR severity among $S$. aureus-positive AR subjects.

Conclusions: The $20 \%$ prevalence of $S$. aureus in AR patients is not different from that of healthy controls in Thailand, and is similar to other reported rates. No significant associations with AR severity were identified.

Key words: Prevalence, nasal carriage, Staphylococcus aureus, allergic rhinitis patients, healthy controls.

\author{
Affiliations: \\ Division of Rhinology \& Allergy, \\ Department of Otorhinolaryngology, Faculty of Medicine \\ Siriraj Hospital, Mahidol University, Bangkok, Thailand \\ Department of Microbiology, Faculty of Medicine Siriraj Hospital, \\ Mahidol University, Bangkok, Thailand
}

\author{
* Corresponding author: \\ Pongsakorn Tantilipikorn \\ Division of Rhinology \& Allergy, \\ Department of Otorhinolaryngology, Faculty of Medicine \\ Siriraj Hospital, Mahidol University \\ 2 Wanglang Road, Bangkoknoi, Bangkok 10700, Thailand \\ E-mail: pongsakorn.tan@mahidol.ac.th
}

\section{Introduction}

The prevalence of allergic rhinitis (AR) has escalated dramatically in recent years. ${ }^{1}$ The seriousness of the disease has also increased, with a commensurate increase in adverse impact on quality of life. Many recent studies have focused on the relationship between Staphyloccoccus aureus (S. aureus) and allergic diseases, such as asthma, atopic dermatitis, and AR. ${ }^{1-5}$

S. aureus is considered to be normal flora. Approximately $25 \%$ of the populations are S. aureus carriers, and many factors may alter the prevalence of the carrier stage of $S$. aureus, including ethnicity, age, gender, and body site. ${ }^{3,6-8}$ Moreover, $S$. aureus infection can develop into a life-threatening condition depending on the Staphylococcal superantigens (SA) that induce inflammatory immune response. In allergic disease, SA increases the sensitivity to antigen and decreases T-cell response to steroid, which may worsen disease severity. ${ }^{1}$

Several studies have reported on the prevalence of nasal carriage of $S$. aureus in healthy controls and in AR patients. Those studies found the prevalence of nasal carriage of $S$. aureus to be significantly higher among $\mathrm{AR}$ patients compared 
to healthy controls, but no information about the pathogenesis was reported. ${ }^{2,9,10}$ Very little is known about the prevalence of this condition in healthy controls and in $\mathrm{AR}$ patients in Thailand.

Accordingly, the aim of this study was to determine the prevalence of nasal carriage of $S$. aureus in AR patients compared with healthy controls in Thailand. The secondary objective was to identify factors significantly associated with AR severity in patients positive for nasal carriage of $S$. aureus.

\section{Material and methods \\ Study population}

This prospective study enrolled non-AR healthy controls and confirmed AR aged 18-60 years who attended the Division of Rhinology \& Allergy, Department of Otorhinolaryngology, Faculty of Medicine Siriraj Hospital, Mahidol University, Bangkok, Thailand during the June 2013 and December 2013 study period. Siriraj Hospital is Thailand's largest national tertiary referral center. The protocol for this study was approved by the Siriraj Institutional Review Board (SIRB) (COA no. 408/2555 (EC2)), and all participants provided written informed consent.

Participants were classified into either the healthy control group or the AR group. All participants were non-smoking, and all patients had normal anterior and posterior rhinoscopy on physical examination. Healthy control group patients had no history of symptoms suggestive of chronic rhinitis, such as rhinorrhea, sneezing, nasal congestion, and/or itching. AR group patients were confirmed by clinical history of rhinorrhea, sneezing, nasal congestion, and itching for at least one hour on most days, as well as by positive skin prick test for any specific allergen(s). Skin prick test was classified into 5 groups, including HDM (house dust, mite), pets (cat, dog), household insects (cockroach, mosquito, housefly, ant), pollens (Bermuda grass, paragrass, sugar cane, sedge, careless weed, typha, mixed grasses), and molds (Cladosporium, Penicillium, Aspergillus, mixed mold). Subjects meeting one or more of the following criteria were excluded: being a healthcare provider; having comorbid disease(s) (e.g., chronic rhinosinusitis, asthma, and/or atopic rhinitis); previous nasal/sinus operation; and/or, prior use of any antibiotics within four weeks.

\section{Study protocol}

For detection of nasal carriage of $S$. aureus, the specimen was collected from the nasal vestibule, including head of inferior turbinate using nasal swab (rayon-tipped swab, BBL ${ }^{\mathrm{m}}$ CultureSwab $^{\mathrm{Tm}}$, Becton Dickinson, Franklin Lakes, NJ, USA). The contact time was at least 3 seconds using the technique developed from Centers for Disease Control (CDC) guideline, 2000. The specimen was then sent to our laboratory for culture within 1 hour. The laboratory system at our center adheres to the standards of the Clinical and Laboratory Standards Institute (CLSI) 2013. ${ }^{11}$

All AR patients were assessed for disease severity using Allergic Rhinitis and its Impact on Asthma (ARIA) guideline, and visual analog scale (VAS) for evaluation of nasal symptom severity. Quality of life was assessed by two validated questionnaires, including the Thai version of the 36-Item
Short Form Health survey (SF-36), and the Rhinoconjunctivitis Quality of Life questionnaires (RCQ-36). The SF-36 has 8 domains, including physical functioning, role-physical, bodily pain, general health, vitality, social functioning, role-emotional, and mental health. The RCQ-36 also has 8 domains, including rhinitis symptoms, eye symptoms, other symptoms, physical functioning, role limitations, sleep, social functioning, and emotions. The relationship between nasal carriage of S. aureus in AR and AR disease severity was analyzed.

\section{Statistical analysis}

SPSS Statistics version 18 (SPSS, Inc., Chicago, IL, USA) was used to perform all statistical analyses. Quantitative data are presented as mean \pm standard deviation, and qualitative data are described as frequency. T-test and chi-square test were used for quantitative and qualitative data comparison, respectively, between $\mathrm{AR}$ and healthy subjects. A p-value less than 0.05 was regarded as being statistically significant.

\section{Results}

Two hundred participants were enrolled in this study. Subjects were equally divided into the healthy control group or the AR group. In the healthy control group, there were 71 females and 29 males, with a mean age of $41.9 \pm 11.4$ years. In the AR group, there were 63 females and 37 males, with a mean age $40.0 \pm 12.2$ years (Table 1 ).

Table 1. Demographic characteristics compared between allergic rhinitis patients and healthy controls

\begin{tabular}{lccc}
\multicolumn{1}{c}{ Characteristics } & $\begin{array}{c}\text { Allergic } \\
\text { rhinitis } \\
(\mathbf{n}=\mathbf{1 0 0})\end{array}$ & $\begin{array}{c}\text { Healthy } \\
\text { controls } \\
(\mathbf{n}=\mathbf{1 0 0})\end{array}$ & p-value \\
\hline Age (yrs) (mean \pm SD) & $40.0 \pm 12.2$ & $41.9 \pm 11.4$ & 0.26 \\
Gender & 63 & 71 & 0.23 \\
Female, $\mathrm{n}$ & 37 & 29 & \\
\hline Male, $\mathrm{n}$ & & & \\
\hline
\end{tabular}

$p$-value $<0.05$ indicates statistical significance

Abbreviation: SD, standard deviation

\section{Rate of nasal carriage of $S$. aureus}

In the healthy control group, nasal swab cultures were positive for S. aureus in 20 of 100 control subjects. In the AR group, nasal swab cultures were positive for $S$. aureus in 21 of 100 patients. All of the positive cultures were methicillin-sensitive $S$. aureus (MSSA) in both groups. The prevalence of nasal carriage of $S$. aureus was not different between groups. ( $p$ $=0.86)$, (Table 2).

Table 2. Nasal carriage of S. aureus compared between allergic rhinitis patients and healthy controls

\begin{tabular}{lccc}
\multicolumn{1}{c}{ S. aureus culture } & $\begin{array}{c}\text { Allergic } \\
\text { rhinitis } \\
(\mathbf{n}=\mathbf{1 0 0})\end{array}$ & $\begin{array}{c}\text { Healthy } \\
\text { controls } \\
(\mathbf{n}=\mathbf{1 0 0})\end{array}$ & p-value \\
Positive, $\mathrm{n}$ & 79 & 80 & 0.86 \\
Negative, $\mathrm{n}$ & 21 & 20 & \\
\hline
\end{tabular}

A $p$-value $<0.05$ indicates statistical significance

Abbreviation: S. aureus, Staphylococcus aureus 
Factors influencing nasal carriage of $S$. aureus in the $A R$ group

Age - Mean age among those with negative S. aureus culture and positive $S$. aureus culture was $41.2 \pm 11.7$ years and $35.5 \pm 13.3$ years, respectively. Nasal carriage was not relevant to age in the AR group.

Gender - Among the 63 females, cultures were negative in 55 patients and positive in 8 patients. Among the 37 males, cultures were negative in 24 patients and positive in 13 patients. The nasal carriage of $S$. aureus in male was statistically significant difference from female $(p=0.01)$, (Table 3).

Table 3. Demographic characteristics compared between negative and positive nasal carriage of $S$. aureus in the allergic rhinitis group

\begin{tabular}{|c|c|c|c|c|}
\hline Characteristics & $\mathbf{n}$ & $\begin{array}{l}\text { Negative } \\
\text { S. aureus } \\
(\mathbf{n}=79)\end{array}$ & $\begin{array}{l}\text { Positive } \\
\text { S. aureus } \\
(\mathbf{n}=21)\end{array}$ & $p$-value \\
\hline Age $($ yrs $)($ mean \pm SD) & & $41.2 \pm 11.7$ & $35.5 \pm 13.3$ & 0.06 \\
\hline $20-29, \mathrm{n}$ & 26 & 16 & 10 & \\
\hline $30-39, \mathrm{n}$ & 20 & 16 & 4 & \\
\hline $40-49, \mathrm{n}$ & 27 & 24 & 3 & \\
\hline $50-60, \mathrm{n}$ & 27 & 23 & 4 & \\
\hline Gender & & & & 0.01 \\
\hline Female, $\mathrm{n}$ & 63 & 55 & 8 & \\
\hline Male, $\mathrm{n}$ & 37 & 24 & 13 & \\
\hline
\end{tabular}

A $p$-value $<0.05$ indicates statistical significance

Abbreviations: S. aureus, Staphylococcus aureus; SD, standard deviation

\section{Type of allergen (Table 4):}

HDM - In patients with positive SPT for HDM, 72/92 patients had negative culture and 20/92 had positive culture. In patients with negative SPT for HDM, 7/8 patients had negative culture and $1 / 8$ had positive culture.

Pets - In patients with positive SPT for pets, 49/65 patients had negative culture and 16/65 had positive culture. In patients with negative SPT for pets, 30/35 patients had negative culture and $5 / 35$ had positive culture.

Household insects - In patients with positive SPT for household insects, 57/74 patients had negative culture and $17 / 74$ had positive culture. In patients with negative SPT for household insects, 22/26 patients had negative culture and $4 / 26$ had positive culture.

Pollens - In patients with positive SPT for pollens, $67 / 84$ patients had negative culture and 17/84 had positive culture. In patients with negative SPT for pollens, 12/16 had negative culture and 4/16 had positive culture.

Molds - In patients with positive SPT for molds, 13/17 patients had negative culture and $4 / 17$ had positive culture. In patients with negative SPT for molds, 66/83 patients had negative culture and $17 / 83$ positive culture.

There was no statistical significance between the nasal carriage of $S$. aureus and the type of positive SPT allergen in both groups.
Table 4. Skin prick test results compared between negative and positive nasal carriage of $S$. aureus in the allergic rhinitis group

\begin{tabular}{|c|c|c|c|c|}
\hline Skin prick test & $\mathbf{n}$ & $\begin{array}{c}\text { Negative } \\
\text { S. aureus } \\
(\mathrm{n}=79)\end{array}$ & $\begin{array}{l}\text { Positive } \\
\text { S. aureus } \\
(\mathrm{n}=21)\end{array}$ & $p$-value \\
\hline HDM & & & & 1 \\
\hline Negative, $\mathrm{n}$ & 8 & 7 & 1 & \\
\hline Positive, $\mathrm{n}$ & 92 & 72 & 20 & \\
\hline Pets & & & & 0.22 \\
\hline Negative, $\mathrm{n}$ & 35 & 30 & 5 & \\
\hline Positive, $\mathrm{n}$ & 65 & 49 & 16 & \\
\hline Household insects & & & & 0.41 \\
\hline Negative, $\mathrm{n}$ & 26 & 22 & 4 & \\
\hline Positive, $\mathrm{n}$ & 74 & 57 & 17 & \\
\hline Pollens & & & & 0.74 \\
\hline Negative, $\mathrm{n}$ & 16 & 12 & 4 & \\
\hline Positive, $\mathrm{n}$ & 84 & 67 & 17 & \\
\hline Molds & & & & 0.75 \\
\hline Negative, $\mathrm{n}$ & 83 & 66 & 17 & \\
\hline Positive, $\mathrm{n}$ & 17 & 13 & 4 & \\
\hline
\end{tabular}

A $p$-value $<0.05$ indicates statistical significance

Abbreviation: S. aureus, Staphylococcus aureus; HDM, house dust, mite

Nasal carriage of $S$. aureus and severity of allergic rhinitis disease

Visual analog scale (VAS) - The mean score was $4.1 \pm 2.6$ among negative culture patients, and $4.3 \pm 2.4$ among positive culture patients. There was no statistical significance in the two groups.

ARIA criteria - Patients was classified into 4 groups according to the ARIA guideline, as follows:

Mild intermittent - Culture was positive in 10/44 patients, and negative in 34/44 patients.

Moderate to severe intermittent - Culture was positive in $3 / 17$ patients, and negative in $14 / 17$ patients.

Mild persistent - Culture was positive in 5/20 patients, and negative in $15 / 20$ patients.

Moderate to severe persistent - Culture was positive in $3 / 19$ patients, and negative in $16 / 19$ patients.

There was no statistical significance between the nasal carriage of $S$. aureus and the severity of allergic rhinitis evaluated by ARIA Criteria.

The 36-Item Short Form Health Survey (SF-36) Thai-version - There was no statistically significant difference between the severity of AR and nasal carriage of $S$. aureus. ( $p$-value range: 0.08-0.95), (Table 5). 
Table 5. Mean \pm standard deviation SF-36 scores compared between negative and positive nasal carriage of $S$. aureus in the allergic rhinitis group

\begin{tabular}{|lccc|}
\hline \multicolumn{1}{|c}{ SF-36 domains } & $\begin{array}{c}\text { Negative } \\
\text { S. Aureus } \\
(\mathbf{n}=\mathbf{7 9})\end{array}$ & $\begin{array}{c}\text { Positive } \\
\text { S. aureus } \\
(\mathbf{n}=\mathbf{2 1})\end{array}$ & p-value \\
\hline Physical functioning & $77.5 \pm 17.4$ & $79.3 \pm 22.9$ & 0.70 \\
\hline Role-physical & $69.6 \pm 40.4$ & $69.0 \pm 35.3$ & 0.95 \\
\hline Bodily pain & $64.8 \pm 18.9$ & $71.7 \pm 23.6$ & 0.16 \\
General health & $45.4 \pm 17.9$ & $49.6 \pm 18.9$ & 0.35 \\
\hline Vitality & $58.2 \pm 15.8$ & $58.6 \pm 14.3$ & 0.92 \\
Social functioning & $72.6 \pm 20.4$ & $79.2 \pm 17.8$ & 0.18 \\
\hline Role-emotional & $68.4 \pm 42.7$ & $82.5 \pm 29.1$ & 0.08 \\
\hline Mental health & $65.3 \pm 16.9$ & $67.4 \pm 13.4$ & 0.59 \\
\hline
\end{tabular}

A $p$-value $<0.05$ indicates statistical significance

Abbreviations: SF-36, 36-Item Short Form Survey; S. aureus, Staphylococcus aureus

Rhinoconjunctivitis Quality of Life questionnaires (RCQ36) Thai-version - There was no statistical significance between the severity of $\mathrm{AR}$ and nasal carriage of $S$. aureus. ( $p$-value range: 0.23-0.92), (Table 6).

Table 6. Mean \pm standard deviation RCQ-36 scores compared between negative and positive nasal carriage of $S$. $a u$ reus in the allergic rhinitis group

\begin{tabular}{|lccc}
\hline \multicolumn{1}{|c}{ RCQ-36 domains } & $\begin{array}{c}\text { Negative } \\
\text { S. aureus } \\
(\mathbf{n}=\mathbf{7 9})\end{array}$ & $\begin{array}{c}\text { Positive } \\
\text { S. aureus } \\
(\mathbf{n}=\mathbf{2 1})\end{array}$ & p-value \\
\hline Rhinitis symptoms & $64.6 \pm 22.5$ & $64.0 \pm 22.3$ & 0.92 \\
\hline Eye symptoms & $71.1 \pm 22.3$ & $75.3 \pm 20.9$ & 0.44 \\
\hline Other symptoms & $70.7 \pm 18.3$ & $76.2 \pm 18.1$ & 0.23 \\
\hline Physical functioning & $78.9 \pm 22.4$ & $77.4 \pm 20.4$ & 0.78 \\
\hline Role limitations & $82.8 \pm 22.6$ & $84.5 \pm 16.7$ & 0.75 \\
\hline Sleep & $73.5 \pm 25.2$ & $78.6 \pm 21.7$ & 0.40 \\
\hline Social functioning & $78.9 \pm 24.3$ & $84.5 \pm 18.3$ & 0.33 \\
\hline Emotions & $66.8 \pm 25.5$ & $70.7 \pm 20.8$ & 0.52 \\
\hline
\end{tabular}

A $p$-value $<0.05$ indicates statistical significance

Abbreviations: RCQ-36, Rhinoconjunctivitis. Quality of Life questionnaire; $S$. aureus, Staphylococcus aureus

All of the 4 severity assessment tools suggested that there was no statistical significance between the severity of allergic rhinitis and the nasal carriage of $S$. aureus.

\section{Healthy controls group}

Age - Mean age was $41.9 \pm 11.6$ years among those with negative $S$. aureus culture, and $42.1 \pm 10.9$ years among those with positive $S$. aureus culture.
Gender - Among the 71 female controls, cultures were negative in 56 women, and positive in 15 women. Among the 29 male controls, cultures were negative in 24 men, and positive in 5 men.

Thus, nasal carriage was not relevant to age and sex in the healthy control group.

\section{Discussion}

Many recent studies have focused on the relationship between $S$. aureus and allergic diseases, such as asthma, atopic dermatitis, and AR..$^{1-5}$ Many factors are likely to alter the prevalence of the carrier stage of $S$. aureus, including ethnicity, age, gender, and body site..$^{6-8,12,13}$ Even though $S$. aureus is regarded as being normal flora, infection by $S$. aureus can be develop into a life-threatening condition. Infection capability and severity depends on the Staphylococcal superantigens that induce inflammatory immune response. In allergic disease, SA increases the sensitivity to antigen and decreases T-cell response to steroid, which may worsen disease severity. ${ }^{1,2} \mathrm{Sev}-$ eral factors have been described as causing an increase in the nasal carriage rate among AR patients. First, inflamed nasal mucosa in AR patients may provide better nutrients. ${ }^{14}$ Second, nasal symptoms, such as rhinorrhea and sneezing, could increase finger to nose contact.

The worldwide nasal carriage rate of $S$. aureus is approximately $20-30 \%$. Among Asian countries, the carriage rate is 23\% in China, 35.7\% in Japan, $9.1 \%$ in Pakistan, $23.4 \%$ in Malaysia, $24.1 \%$ in Taiwan, and $29.4 \%$ in India. ${ }^{8} 12$ The two studies conducted in Thailand, both of which had study populations consisting of university students, found prevalence rates of $15.0 \%$ and $27.7 \%$, respectively. ${ }^{15,16}$ The carriage rate in our study was approximately $20 \%$ among healthy controls, which is similar to the rate worldwide and in Asia.

The nasal carriage rate of $S$. aureus among AR patients in this study was not significantly higher than the rate among healthy controls ( $21 \% v s, 20 \%$, respectively), which was inconsistent with previous studies. ${ }^{2,9,10}$ Riechelmann, et al. ${ }^{2}$ and Shiomori, et al. ${ }^{9}$ reported the prevalence of nasal carriage of $S$. aureus in AR patients to be higher than in the control group. Moreover and in contrast to our study, the nasal symptom score in those studies was significantly higher in positive culture patients than in negative culture patients.

The difference in the prevalence of nasal carriage of $S$. aureus between the present study and other studies can be explained by the nasal carriage pattern. It may be persistent or intermittent carriage. Still, there is no consensus regarding how many cultures should be taken to define persistent carriage. Specimen collection could also be another factor. The study of Riechelmann used the nasal lavages to assess the number of SA. ${ }^{2}$ They discussed about the semi quantitative assessment of nasal SA colonization. But the pathophysiology of SA for allergic disease is the 'intracellular' SA, which acts as the superantigen. ${ }^{5}$ This is the possibility of explanation of contradiction. Another hypothesis is the possibility of difference strain of SA of production of SA super antigen between Asia and European. Timing and techniques (nasal lavage versus nasal swab culture) might affect the yield of positive cultures. 
$S$. aureus detection by polymerase chain reaction (PCR) would increase the sensitivity for detection of $S$. aureus; however, we used culture, which is the standard technique, ${ }^{17}$ in our study.

Data relating to saline solution (NSS) irrigation for treatment of AR, and type and intermittent use of previous medications were not collected in this study. NSS irrigation could decrease viral load before specimen collection. Antibiotics, such as rifampicin, doxycyclin, and topical mupirocin ointment, could eliminate the nasal carriage stage of $S$. aureus. ${ }^{17}$ These factors and others could have confounding effects on our results. In contrast, intranasal corticosteroid was reported to have no effect on the prevalence of nasal carriage of $S$. aureus. ${ }^{14,18}$

Our findings revealed males to be more likely to have nasal carriage of $S$. aureus than females. A study from Iran also found male predominance. ${ }^{19}$ The other study did not show any sex influence on the SA carriage status. ${ }^{20}$ The significance of this clinical characteristic needs to be further elucidated. Moreover, we found no significant association between other factors that might influence disease severity among patients positive for nasal carriage of $S$. aureus. The severity of AR was not significantly different between those that did and that didn't carry S. aureus. Importantly, we did not classify AR patients into seasonal and perennial allergic rhinitis. Instead, we classified patients according to the ARIA guideline, since it is more suitable for use in Thailand.

The novel method of determining status of S. aureus or microbiome status can be the trend of future studies. Staphyloccoccal superantigens likely play an important role in the pathogenesis of AR. Moreover, they are relevant to the nasal carriage stage. Techniques of determining the diversity index of nasal microbiome are currently ongoing to define the pathophysiology of persistent nasal inflammation.

\section{Conclusion}

The prevalence of $S$. aureus in allergic rhinitis patients was not different from healthy controls in Thailand. The nasal carriage rate in healthy controls was about $20 \%$, similar to the worldwide studies. No significant associations were found between evaluated factors and AR severity in AR patients with nasal carriage of $S$. aureus.

\section{Acknowledgments}

The authors gratefully acknowledge the patients that generously agreed to participate in this study, Mr. Suthipol Udompunthurak of the Division of Clinical Epidemiology, Research Department, Faculty of Medicine Siriraj Hospital, Mahidol University for assistance with statistical analysis, and Ms. Jeerapa Kerdnoppakhun for assistance with manuscript development.

\section{Conflict of interest declaration}

All authors declare no personal or professional conflicts of interest, and no financial support from the companies that produce and/or distribute the drugs, devices, or materials described in this report.

\section{Funding disclosure}

This study was funded by a grant from the Faculty of Medicine Siriraj Hospital Research Fund.

\section{References}

1. Pastacaldi C, Lewis P, Howarth P. Staphylococci and staphylococcal superantigens in asthma and rhinitis: a systematic review and meta -analysis. Allergy. 2011;66(4):549-55.

2. Riechelmann H, Essig A, Deutschle T, Rau A, Rothermel B, Weschta M. Nasal carriage of Staphylococcus aureus in house dust mite allergic patients and healthy controls. Allergy. 2005;60(11):1418-23.

3. Bachert C, Gevaert P, van Cauwenberge P. Staphylococcus aureus superantigens and airway disease. Curr Allergy Asthma Rep. 2002;2(3): 252-8.

4. Bachert C, Gevaert P, Zhang N, van Zele T, Perez-Novo C. Role of staphylococcal superantigens in airway disease. Chem Immunol Allergy. 2007;93:214-36.

5. Stow NW, Douglas R, Tantilipikorn P, Lacroix JS. Superantigens. Otolaryngol Clin North Am. 2010;4(3):489-501.

6. Choi CS, Yin CS, Bakar AA, Sakewi Z, Naing NN, Jamal F, et al. Nasal carriage of Staphylococcus aureus among healthy adults. J Microbiol Immunol Infect. 2006;39(6):458-64.

7. Kluytmans J, van Belkum A, Verbrugh H. Nasal carriage of Staphylococcus aureus: epidemiology, underlying mechanisms, and associated risks. Clinical Microbiology Reviews. 1997;10(3):505-20.

8. Sollid JU, Furberg AS, Hanssen AM, Johannessen M. Staphylococcus aureus: Determinants of human carriage. Infect Genet Evol. 2014;21: 531-41.

9. Shiomori T, Yoshida S, Miyamoto H, Makishima K. Relationship of nasal carriage of Staphylococcus aureus to pathogenesis of perennial allergic rhinitis. Journal of Allergy \& Clinical Immunology. 2000;105(3):449-54.

10. Refaat MM, Ahmed TM, Ashour ZA, Atia MY. Immunological role of nasal staphylococcus aureus carriage in patients with persistent allergic rhinitis. Pan Afr Med J. 2008;1:3.

11. Clinical and Laboratory Standards Institute. Performance Standards for Antimicrobial Susceptibility Testing; Twenty-Fourth Informational Supplement. CLSI document M100-S24. Pennsylvania: Clinical and Laboratory Standards Institute; 2014.

12. Wertheim HF, Melles DC, Vos MC, van Leeuwen W, van Belkum A, Verbrugh HA, et al. The role of nasal carriage in Staphylococcus aureus infections. Lancet Infect Dis. 2005;5(12):751-62.

13. Halablab MA, Hijazi SM, Fawzi MA, Araj GF. Staphylococcus aureus nasal carriage rate and associated risk factors in individuals in the community. Epidemiol Infect. 2010;138(5):702-6.

14. Baysoy G, Arslan S, Karabay O, Uyan AP. Nasal carriage of Staphylococcus aureus in children with allergic rhinitis and the effect of intranasal fluticasone propionate treatment on carriage status. Int J Pediatr Otorhinolaryngol. 2007;71(2):205-9.

15. Treesirichod A, Hantagool S, Prommalikit O. Nasal carriage and antimicrobial susceptibility of Staphylococcus aureus among medical students at the HRH Princess Maha Chakri Sirindhorn Medical Center, Thailand: a cross sectional study. J Infect Public Health. 2013;6(3):196-201.

16. Kitti T, Boonyonying K, Sitthisak S. Prevalence of methicillin-resistant Staphylococcus aureus among university students in Thailand. Southeast Asian J Trop Med Public Health. 2011;42(6):1498-504.

17. Lucet JC, Regnier B. Screening and decolonization: does methicillin -susceptible Staphylococcus aureus hold lessons for methicillin-resistant S. aureus? Clin Infect Dis. 2010;51(5):585-90.

18. Bunnag C, Vipulakom P, Pacharee P, Siriyananda C. Intranasal inhalation of beclomethasone dipropionate in the treatment of perennial rhinitis in adults. Ann Allergy. 1980;44(2):100-5.

19. Nasiri B, Ghotaslou R, Ballali L, Darbin A. Prevalence of Nasal Carriage of Staphylococcus aureus in Madani Heart Hospital, Tabriz. J Cardiovasc Thorac Res. 2010;2(3):13-7.

20. Okano M, Takishita T, Yamamoto T, Hattori H, Yamashita Y, Nishioka S. et al. Presence and Characterization of Sensitization to Staphylococcal Enterotoxins in Patients with Allergic Rhinitis. Am J Rhinol. 2001;15: 417-21. 